\title{
Laparoscopic surgical technique to facilitate management of high anorectal malformations - report of seven cases
}

\author{
Andrzej Gołębiewski, Maciej Murawski, Marcin Łosin, Marek Królak, Piotr Czauderna \\ Department of Surgery and Urology for Children and Adolescents, Medical University, Gdansk, Poland
}

Videosurgery and other miniinvasive techniques 2011; 6 (3): 150-154 DOI: 10.5114/wiitm.2011.24693

\begin{abstract}
Anorectal malformations (ARMs) occur in approximately 1 per 5000 live births. The most commonly used procedure for repair of high ARMs is posterior sagittal anorectoplasty (PSARP). This operation is performed entirely through a perineal approach. The first report of laparoscopically assisted anorectal pull-through (LAARP) for repair of ARMs was presented by Georgeson in 2000. The aim is presenting early experience with laparoscopically assisted anorectal pull-through technique in boys with high anorectal malformations. In the last 5 years 7 boys ( 9 months to 2 years old) with high ARMs were operated on using the LAARP technique. Laparoscopically the rectal pouch was exposed down to the urethral fistula, which was clipped and divided. Externally, the centre of the muscle complex was identified using an electrical stimulator. In the first 4 patients after a midline incision of $2 \mathrm{~cm}$ at the planned anoplasty site, a tunnel to the pelvis was created bluntly and dilated with Hegar probes under laparoscopic control. In the last 3 boys a minimal PSARP was done creating a channel into the pelvis. The separated rectum was pulled down and sutured to the perineum. Laparoscopic mobilization of the rectal pouch and fistula division was possible in all cases. There were no intraoperative complications except one ureteral injury. Patients were discharged home on post-operative day 5 to 7. The early results prove that $L A A R P$, an alternative option to PSARP for treatment of imperforate anus, offers many advantages, including excellent visualization of the pelvic anatomical structures, accurate placement of the bowel into the muscle complex and a minimally invasive abdominal and perineal incision. It allows for shorter hospital stay and faster recovery. However, to compare the functional results against the standard procedure (PSARP), longer followup of all patients is necessary.
\end{abstract}

Key words: laparoscopy, anorectal malformations, laparoscopically assisted anorectal pull-through (LAARP), children.

\section{Introduction}

Anorectal malformations (ARMs) are common congenital abnormalities, occurring in approximately 1 in 5000 live births with male predominance. They represent a spectrum of congenital defects, sometimes with associated intestinal, renal, cardiac, vertebral and limb anomalies (VACTERL association). They are subdivided into low and high defects depending on the location of the rectal pouch. In high defects the rectal pouch terminates above the levator muscles, while in low ARMs the rectal pouch passes through the levators. Low defects are treated during the neonatal period with primary anoplasty without colostomy. High defects, which account for nearly $80 \%$ of male ARMs, require a diverting colostomy in the newborn period and definitive repair usually by 3-6 months of age. These malformations are usually treated with posterior sagittal anorectoplasty (PSARP) according to deVries and Peña [1, 2].

Posterior sagittal anorectoplasty is performed in the prone position and a mid-sagittal incision from the 
coccyx to the perineal body is used to divide the subcutaneous tissue, parasagittal fibres, muscle complex, and levator muscle equally along the midline. After opening the levator muscles, the rectum is found and opened in the midline to demonstrate presence of fistula to the urinary tract. The fistula is separated from the urethra and ligated with absorbable suture. In 10\% of patients with a fistula to the bladder neck laparotomy is additionally required to ligate the fistula and to mobilize the rectum off the bladder neck. Once the rectum is separated it is then mobilized down to reach the peritoneum. The rectum is placed within the muscle complex and sutured to the perineal skin (anoplasty). Nowadays it is possible to perform this procedure by minimally invasive surgical techniques. Willital in 1998 [3] was the first to describe the use of laparoscopy in the repair of ARMs. Georgeson afterwards popularized the technique and called it laparoscopically assisted anorectal pull-through (LAARP) [4]. He realized that laparoscopy could offer improved exposure for dissection of the fistula and facilitate rectal mobilization without the need for extensive perineal surgery. Since then LAARP has increased its popularity among paediatric surgeons and in many cases replaced standard open procedures.

\section{Case reports}

This is a retrospective consecutive case series analysing LAARP performed in seven boys with high anorectal malformations since 2005. The age at operation ranged from 9 months to 2 years (average 15 months). All patients were treated initially with divided sigmoid colostomy at birth for high imperforate anus. Before the procedure all underwent distal colostograms to define the site of communication of rectal fistulae with genitourinary structures. Clinical characteristics of the patients are presented in Table I.

Laparoscopy was performed as described by Georgeson et al. [4]. After bowel preparation intravenous antibiotic was given before surgery. The child was placed in a supine position and the urinary bladder was catheterized. After antiseptic skin preparation from the thorax to the knees, the first $5 \mathrm{~mm}$ trocar was inserted in an open fashion through the inferior umbilical fold. After creating pneumoperitoneum of the $\mathrm{CO}_{2}$ pressure of 6 to $10 \mathrm{mmHg}$, two additional $5 \mathrm{~mm}$ trocars were placed in the right and left upper quadrant (another option for port placement was the midline above the umbilicus and to the left of the umbilicus). Sometimes an additional $3 \mathrm{~mm}$ instrument was introduced through the stab wound in the right hypogastrium or a stay suture was placed suprapubically to help retract the bladder. The $30^{\circ}$ scope placed through an umbilical port was used.

Laparoscopic rectal dissection was begun at the peritoneal reflection. Using hook electrocautery the distal mesorectum was divided and dissection continued anteriorly and laterally on the rectal wall (Figure 1). The terminal branches of the sigmoid and

Table I. Patients' characteristics

\begin{tabular}{|c|c|c|c|c|}
\hline Patient No. & $\begin{array}{l}\text { Age at LAARP } \\
\text { [months] }\end{array}$ & Rectourinary fistula & VACTERL & $\begin{array}{l}\text { Colostomy } \\
\text { at birth }\end{array}$ \\
\hline 1 & 22 & Bulbar urethra & Left kidney agenesis, VSD, bilateral cryptorchidism & Yes \\
\hline 2 & 10 & Prostatic urethra & None & Yes \\
\hline 3 & 24 & Bladder neck & $\begin{array}{l}\text { Crossed left kidney ectopia, right kidney agenesis, } \\
\text { hypospadias, cryptorchidism }\end{array}$ & Yes \\
\hline 4 & 12 & Prostatic urethra & $\begin{array}{l}\text { Left kidney agenesis, cryptorchidism, } \\
\text { Meckel's diverticulum, }\end{array}$ & Yes \\
\hline 5 & 17 & Bulbar urethra & Sacral bone hypoplasia & Yes \\
\hline 6 & 11 & Bulbar urethra & $\begin{array}{l}\text { Bilateral dysplastic kidneys, renal insufficiency, } \\
\text { VUR grade } 5 \text { on left }\end{array}$ & Yes \\
\hline 7 & 9 & Prostatic urethra & None & Yes \\
\hline
\end{tabular}

VSD - ventricular septal defect, VUR - vesicoureteral reflux 
superior rectal arteries were divided to gain adequate bowel length. During dissection special care was taken not to injure ureters and surrounding genital structures. As the fistula was identified it was followed down to its communication with the urethra and clipped with HemoLocks (Figure 2) as close to the urethra as possible and then sharply divided (Figure 3 ). After retraction of the colon the pelvic floor was inspected and the pubococcygeus muscle was identified. Next the legs were elevated and the perineum exposed. Using the muscle stimulator the sphincteric complex was identified and the optimal place for the anoplasty was determined. In the first 4 patients a midline incision of $2 \mathrm{~cm}$ at the planned anoplasty site was done. A tunnel to the pelvis through the muscle complex was created bluntly under laparo-

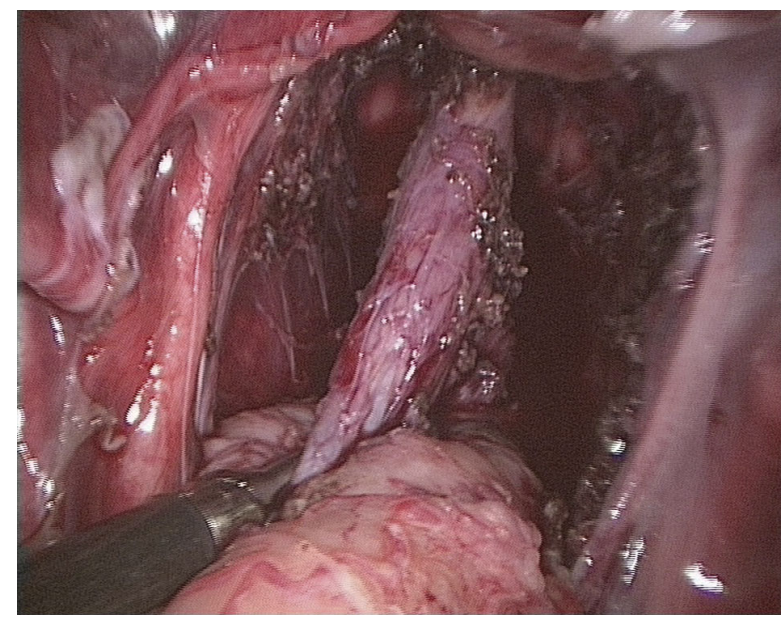

Figure 1. Separated distal rectum with fistula to prostatic urethra

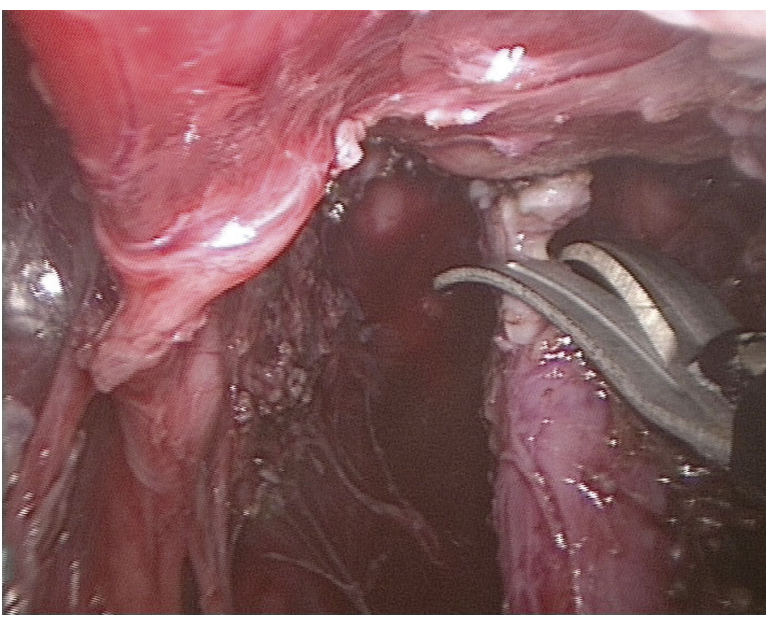

Figure 3. Dissection of fistula between clips scopic guidance and dilated gently in stepwise fashion with Hegar probes up to $10 \mathrm{~mm}$. In the last three boys after dividing the fistula a minimal PSARP was done under electrostimulation control, creating a passage into the pelvis. The separated rectum was grasped and pulled down through the muscle complex to the perineum. Anoplasty was performed with absorbable interrupted 4-0 sutures (Figure 4).

Seven boys with high ARMs treated with initial colostomy in the newborn period were operated on using the laparoscopic approach at the age of 9 months to 2 years (average 15 months). Laparoscopic mobilization of the rectal pouch and fistula division was possible in all cases. There were no intraoperative complications except one of the first cases in which the ureter was accidentally transected. In

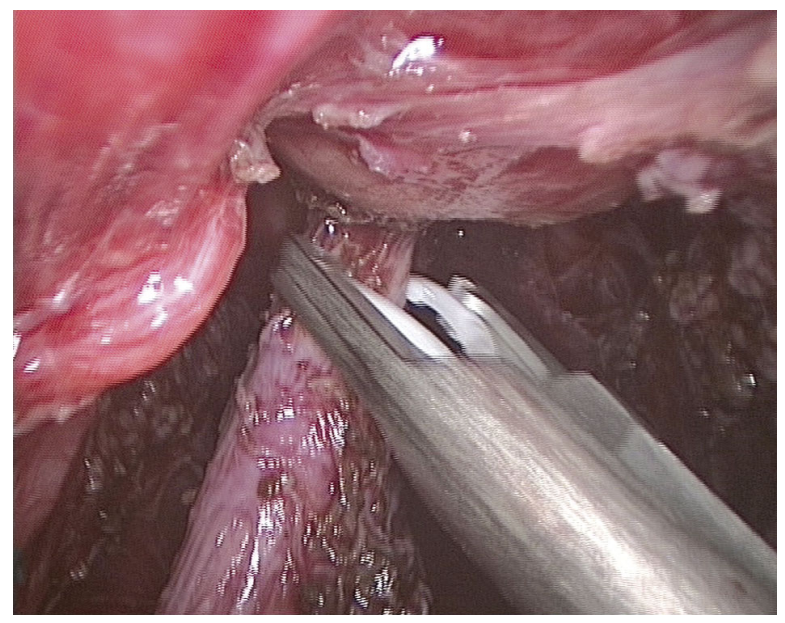

Figure 2. Clipping of fistula

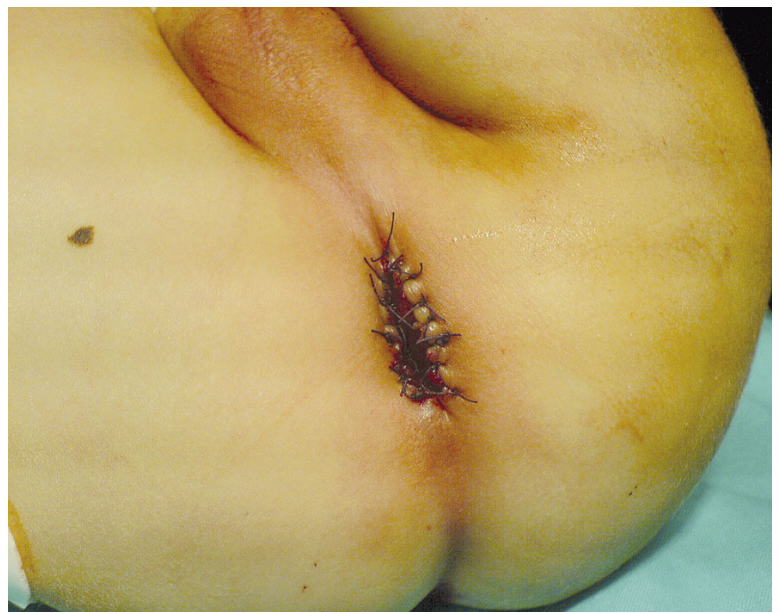

Figure 4. Final reconstruction of anus 
this boy during dissection of the mesorectum the left ureter was injured. The damage was suspected at the time of the operation, but only confirmed during the first postoperative day. Since an attempt to catheterize the ureter by cystoscopy failed, laparotomy was performed; the injured portion of the ureter was excised and re-anastomosed using a double-J stent. The patient underwent reoperation on the $20^{\text {th }}$ postoperative day due to mechanical ileus. In other patients the postoperative course was uneventful except one patient with wound infection treated conservatively. Patients were discharged home on postoperative day 5 to 7 (on the $22^{\text {nd }}$ day in the case of the patient with ureteral injury). The last operated patient with chronic renal failure was transferred to the nephrology department on the second postoperative day.

\section{Discussion}

Posterior sagittal anorectoplasty popularized by deVries and Peña has been a standard surgical procedure for management of imperforate anus for more than 20 years. According to Peña adequate visualization of the sphincteric complex can be achieved through the posterior sagittal approach. Midline division of the muscles with the aid of electrical stimulation allows for identification of a urinary fistula and mobilization of the bowel. However, there is still a controversy regarding the need for a wide perineal incision and total division of the sphincteric complex necessary for proper visualization of a fistula and adequate mobilization of the rectum. Some authors believe it can lead to sphincteric incompetence. Also keeping the dissection in the proper plane can be challenging even for experienced surgeons [5]. Moreover, at least $10 \%$ of patients with high ARMs and rectovesical fistula need laparotomy because this type of fistula cannot be reached from the perineum. In view of these factors the benefits of LAARP include alleviation of laparotomy and minimal surgical trauma to the continence mechanism (e.g. pelvic nerves and muscles) and potentially fewer wound complications.

Repair of ARMs and in particular separation of the rectum and urinary tract creates a major risk of injury to the genitourinary tract including the urethra, bladder, ureters, vas deferens, seminal vesicle, prostate and pelvic nerves. Hong et al. found such complications in $13 \%$ of boys who underwent PSARP [6].
According to his conclusion there is a significant risk of urological injuries associated with the repair of ARMs. Most of them could be prevented with the proper preoperative examination, especially distal colostogram to show the level of the urinary fistula. Reports regarding urological complications after LAARP are rare, mostly involving residual diverticulum and urethral injury [7]. Although Georgeson recommends special attention during division of the mesorectum to avoid injury to the left ureter, such a complication occurred during operation of our second case. It was due to difficult anatomical conditions: ectopic ureters run together with deferent ducts immediately adjacent to the fistula having a common entrance to the bladder. We believe that this complication was related to the learning curve. No urological complications occurred in further operated patients. The change of the perineal approach to minimal PSARP was undertaken due to the report on unsatisfactory functional results with dilatation of the tunnel through the sphincteric complex, presented during the EUPSA Congress in 2009 [8]. We have to admit that to our knowledge the presented series is the biggest in Poland.

\section{Conclusions}

According to our limited experience laparoscopic assistance of the pull-through for imperforate anus offers many advantages, including excellent visualization of the anatomical structures of the pelvis and rectal fistula, which is often superior to that achieved with open surgery. Laparoscopically assisted anorectal pull-through allows for exact placement of the bowel through the muscle complex. This technique avoids the need for laparotomy and an extensive perineal dissection. Finally it results in shorter hospital stay and faster recovery. However, long-term followup is necessary for complete evaluation of functional results and their comparison to the standard procedure (PSARP).

\section{References}

1. deVries PA, Peña A. Posterior sagittal anorectoplasty. J Pediatr Surg 1982; 17: 638-43.

2. Peña A, Devries PA. Posterior sagittal anorectoplasty: important technical considerations and new applications. J Pediatr Surg 1982; 17: 796-811.

3. Willital GH. Endosurgical intrapuborectal reconstruction of high anorectal anomalies. Pediatr Endosurg Innov Tech 1998; 2: 5-11. 
4. Georgeson KE, Inge TH, Albanese CT. Laparoscopically assisted anorectal pull-through for high imperforate anus - a new technique. J Pediatr Surg 2000; 35: 927-30.

5. Piątkowski J, Jackowski M. Laparoscopic colon resection - own experience report. Videosurgery and other miniinvasive techniques 2009; 4: 135-7.

6. Hong AR, Acuña MF, Peña A, et al. Urologic injuries associated with repair of anorectal malformations in male patients. J Pediatr Surg 2002; 37: 339-44.

7. Uchida H, Iwanaka T, Kitano Y, et al. Residual fistula after laparoscopically assisted anorectoplasty: is it a rare problem? J Pediatr Surg 2009; 44: 278-81.

8. Personal communication. 